INPLASY

PROTOCOL

To cite: Li et al. Mean platelet volume and polycystic ovary syndrome: A systematic review and meta-analysis. Inplasy protocol 2021100021. doi: 10.37766/inplasy2021.10.0021

Received: 07 October 2021

Published: 07 October 2021

Corresponding author: Zhongwei Zhou

zzw2858@njmu.edu.cn

Author Affiliation:

Yancheng Third People's Hospital (The Affiliated Yancheng Hospital of Southeast University Medical College, The Sixth Affiliated Hospital of Nantong University, The Yancheng School of Clinical Medicine of Nanjing Medical University).

Support: None.

Review Stage at time of this submission: Completed but not published.

Conflicts of interest: None declared.

\section{Mean platelet volume and polycystic ovary syndrome: A systematic review and meta-analysis}

\author{
Li, L1; Yu, J2; Zhou, Z3.
}

Review question / Objective: Recently, considerable researches measured MPV levels in patients with PCOS to verify whether it is associated with PCOS and its severity. However, inconsistent findings have been achieved on the association between them. To clarify the role of MPV in PCOS, the present systematic review and meta-analysis were conducted on all eligible studies to evaluate the relationship between MPV and PCOS based on evidences.

Condition being studied: Women with PCOS are at an increased risk of developing type 2 diabetes mellitus (T2DM), metabolic syndrome, and cardiovascular disease (CVD). As revealed in recent studies, platelet activation is critical to the pathophysiology of T2DM and CVD. Mean platelet volume (MPV), an inexpensive and easy parameter derived from complete blood cell counts in the daily clinical practice, indicates platelet size and has long acted as an effective marker of platelet activity. Larger platelets exhibiting higher MPV are metabolically and enzymatically more active and produce greater amounts of platelet-activating factors, thereby contributing to the development of CVD.

INPLASY registration number: This protocol was registered with the International Platform of Registered Systematic Review and Meta-Analysis Protocols (INPLASY) on 07 October 2021 and was last updated on 07 October 2021 (registration number INPLASY2021100021).

\section{INTRODUCTION}

Review question / Objective: Recently, considerable researches measured MPV levels in patients with PCOS to verify whether it is associated with PCOS and its severity. However, inconsistent findings have been achieved on the association between them. To clarify the role of MPV in PCOS, the present systematic review and meta-analysis were conducted on all eligible studies to evaluate the relationship 
between MPV and PCOS based on evidences.

Condition being studied: Women with PCOS are at an increased risk of developing type 2 diabetes mellitus (T2DM), metabolic syndrome, and cardiovascular disease (CVD). As revealed in recent studies, platelet activation is critical to the pathophysiology of T2DM and CVD. Mean platelet volume (MPV), an inexpensive and easy parameter derived from complete blood cell counts in the daily clinical practice, indicates platelet size and has long acted as an effective marker of platelet activity. Larger platelets exhibiting higher MPV are metabolically and enzymatically more active and produce greater amounts of platelet-activating factors, thereby contributing to the development of CVD.

\section{METHODS}

Participant or population: Studies were eligible for inclusion if they met the following criteria: (1) studies for adult subjects (age $\geq 18$ years); (2) studies comparing MPV between PCOS women and non-PCOS women; (3) no statistically significant differences between cases and controls in terms of mean age and body mass index (BMI). Studies were discarded when (1) non-English language studies; (2) interventional studies or samples overlapped with another study; or (3) reviews, letters to the editor, case reports, or conference abstracts.

Intervention: MPV was main Exposure/ Interventions.

Comparator: MPV was compared between PCOS women and non-PCOS women.

Study designs to be included: Observational studies that compared MPV between PCOS women and non-PCOS women were included.

Eligibility criteria: (1) studies for adult subjects (age $\geq 18$ years); (2) studies comparing MPV between PCOS women and non-PCOS women; (3) no statistically significant differences between cases and controls in terms of mean age and body mass index (BMI).

Information sources: Systematic literature search was performed in the electronic databases including Pubmed, EMBASE, and Web of Science.

Main outcome(s): Standardized mean difference (SMD) and 95\% confidence interval $(\mathrm{Cl})$ were calculated to estimate the difference in MPV between PCOS cases and controls.

Quality assessment / Risk of bias analysis: Study quality of the included studies was assessed by complying with a modified criterion under the Newcastle-Ottawa Quality Assessment Scale (NOS) for observational studies.

Strategy of data synthesis: A randomeffects model was used in data synthesis process.

Subgroup analysis: Subgroup analysis was conducted by using study design and study location.

Sensitivity analysis: Sensitivity analysis was conducted to assess the stability of the results by sequentially removing one study at a time.

Country(ies) involved: China.

Keywords: Polycystic ovary syndrome, mean platelet volume, meta-analysis.

Contributions of each author:

Author 1 - Li Li.

Author 2 - Jianxiu Yu.

Author 3 - Zhongwei Zhou. 